\title{
Discovering topological surface states of Dirac points
}

\author{
Hengbin Cheng, ${ }^{1,2}$ Yixin Sha, ${ }^{3}$ Rongjuan Liu, ${ }^{1}$ Chen Fang, ${ }^{1,4}$ and Ling $\mathrm{Lu}^{1,4,}$ * \\ ${ }^{1}$ Institute of Physics, Chinese Academy of Sciences/Beijing National \\ Laboratory for Condensed Matter Physics, Beijing 100190, China \\ ${ }^{2}$ School of Physical Sciences, University of Chinese Academy of Sciences, Beijing 100049,China \\ ${ }^{3}$ School of Electronics Engineering and Computer Science, Peking University, Beijing 100871, China \\ ${ }^{4}$ Songshan Lake Materials Laboratory, Dongguan, Guangdong 523808, China.
}

(Dated: January 20, 2020)

\begin{abstract}
Dirac materials, unlike the Weyl materials, have not been found in experiments to support intrinsic topological surface states, as the surface arcs in existing systems are unstable against symmetrypreserving perturbations. Utilizing the proposed glide and time-reversal symmetries, we theoretically design and experimentally verify an acoustic crystal of two frequency-isolated three-dimensional Dirac points with $Z_{2}$ monopole charges and four gapless helicoid surface states.
\end{abstract}

A three-dimensional (3D) Dirac point [1] disperses the same way as the solutions to the massless Dirac equation at the vicinity of the four-fold linear point degeneracy. Playing a central role in 3D band topology, Dirac points can, upon symmetry breaking, transition into Weyl points, line nodes or topological bandgaps with gapless surface states. Although 3D Dirac points have been experimentally discovered in electron 2 7 , magnon 8, 9 and photonic [10] systems along with a variety of other theoretical proposals [11 23, none of the surface states are topological. Specifically, there have been no robust gapless surface bands associated with the bulk Dirac points [24 26].

The current lack of topological surface states for Dirac points can be understood through the anticrossing of two Weyl surface states. Illustrated in Fig. 1, the topological surface dispersion of a Weyl crystal is a doubly-periodic helicoid sheet whose singularities locates at the projection of the bulk Weyl points. The chirality of the helicoid around each Weyl point equals the sign of its Chern number. Since a Dirac point is composed of two Weyl points of opposite Chern numbers, the Dirac surface state should be composed of two helicoids of opposite chiralities. Two opposite helicoid surfaces generally cross each other along a line of momenta and anticross (hybridize with each other), resulting in gapped surface bands which are topologically trivial. The only exception was theoretically proposed in Ref. [27, in which the glide symmetries combined with the time-reversal $(\mathcal{T})$ can stabilize a degenerate line and protect the crossing of the helicoids. As illustrated in Fig. 1, one glide can protect double helicoids and two glides can protect quad-helicoid surfaces states.

In this work, we present an acoustic band structure with two ideal $Z_{2}$ Dirac points protected by glide reflections. The acoustic crystal is 3D-printed and the measured surface dispersions exhibit quad-helicoid surface sheets.

Ideal acoustic Dirac points The cubic cell of the acoustic crystal, in Fig. 2(a), consists of thick rods and thin sticks, belonging to space group $I a \overline{3}$ (No. 206) of the body-centered-cubic (BCC) lattice. The four thick rods of radius $0.15 a$ point at the directions of the $\mathrm{BCC}$ lattice vectors, where $a$ is the lattice constant of the cubic cell. These disconnected rods form the BPI (blue phase I of liquid crystal) photonic crystal in Ref. 28, 29]. We add the thin sticks to connect all rods and mechanically support the whole structure. The sticks are too thin, $0.025 a$ in radius, to change the Dirac acoustic bands, as compared in Supplementary Materials. The background material is air and the interfaces are treated as sound hard-wall boundaries in numerical simulations.

There are two Dirac points locate at the $\pm P$ points of the BCC Brillouin zone [BZ, Fig. 2(b)], where four bands (from the fifth to the eighth in ascending energy order) meet, as shown in Fig. 2(c). The density of states (DOS) [30] vanishes at the Dirac frequency and grows quadratically away from it, as expected for linear dispersion relations.

The local Hamiltonian of this Dirac point is $H(\boldsymbol{k}) \sim$

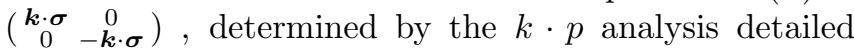
in the Supplementary Materials. This four-fold degeneracy is joined, due to the anti-unitary parity-time symmetry $(\mathcal{P} \mathcal{T})$, by two conjugated $2 \mathrm{D}$ representations of the little group. The little group of No. 206 at $P$ also has a four fold representation which is the generalized Dirac point discussed in Ref. [28, 29]. The Dirac point has identical group velocities while the generalized one does not.

Each Dirac point is stabilized by the products of $\mathcal{T}$ and the three non-commuting glides $\left(G_{x}=\left\{M_{x} \mid(a / 2) \hat{x}+\right.\right.$ $\left.(a / 2) \hat{y}\}, G_{y}=\left\{M_{y} \mid(a / 2) \hat{x}\right\}, G_{z}=\left\{M_{z} \mid(a / 2) \hat{y}\right\}\right)$, denoted as $G_{i} \mathcal{T}(i=x, y, z) . M_{i}$ are the mirror operations and the inversion $\mathcal{P}=G_{x} G_{y} G_{z}$. Each $G_{i} \mathcal{T}$ symmetry enforces a line degeneracy at the zone boundary, represented by the dashed lines in all figures consistently. The three degeneracy lines intersect at the $P$ point, shown in Fig. 2(b), (e). We note that the $P$ point have neither $\mathcal{T}$ nor $G_{i}$ symmetries by themselves.

$Z_{2}$ monopoles Dirac point is the symmetry-protected $Z_{2}$ monopole in the 3D momentum space [31 33. However, the $Z_{2}$ symmetries in the these systems $(\mathcal{P} \mathcal{T}$ for 

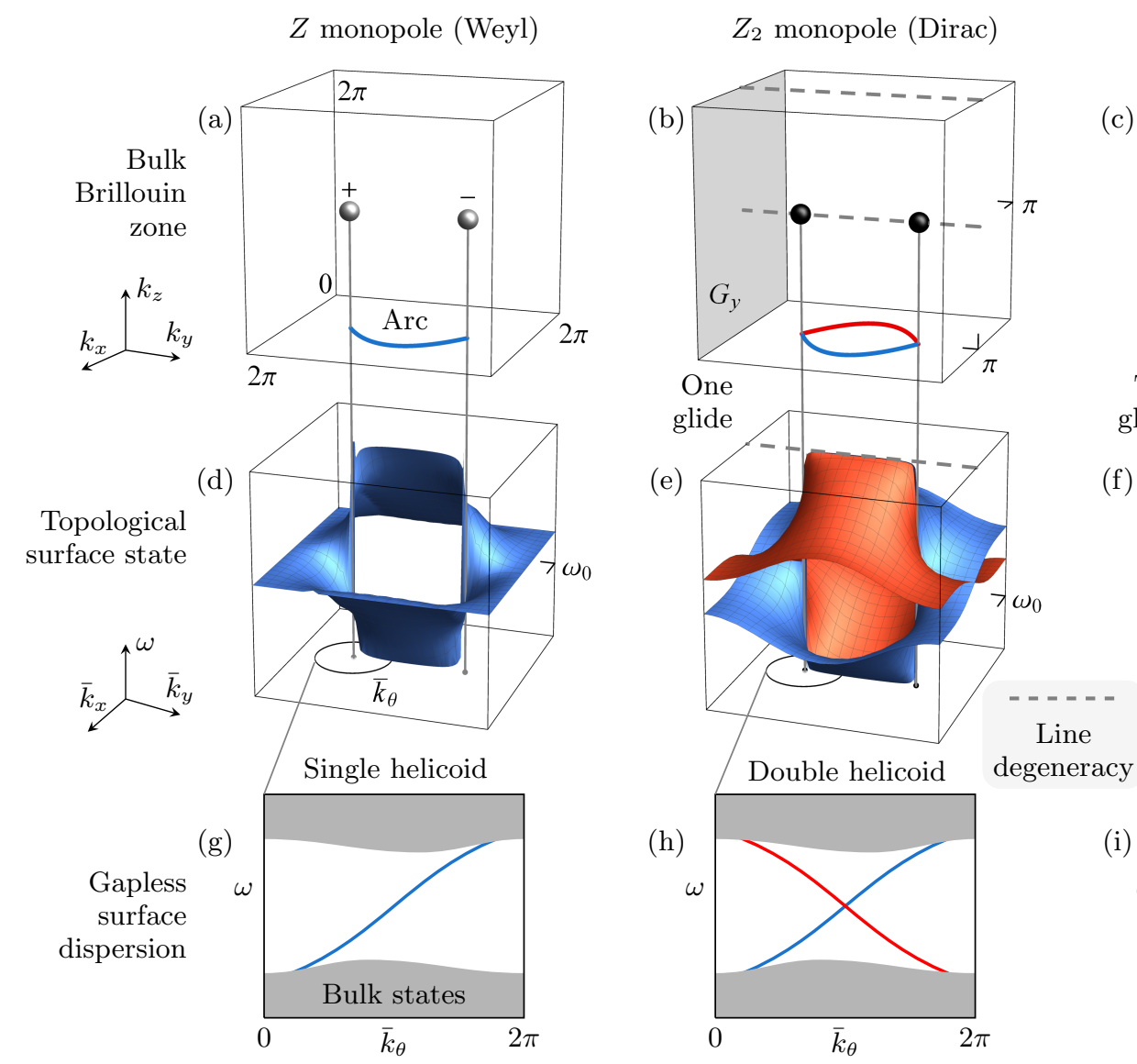

$Z_{2}$ monopoles (Dirac)

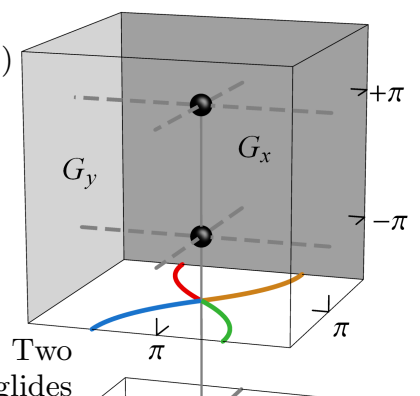

(f)

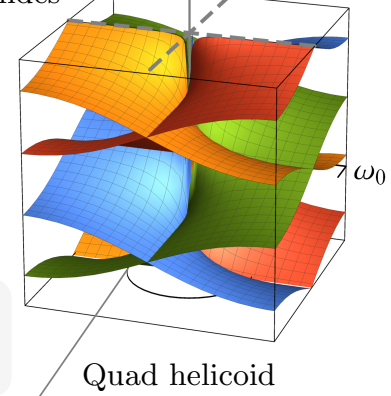

FIG. 1. Bulk monopoles and helicoid surfaces. (a), (b), (c) are the 3D BZ containing two $Z$ or $Z_{2}$ monopoles. (a), (b) can be the BZs of cubic lattices, while (c) is the BZ of the BCC lattice in our acoustic design. The arcs, at the bottom plane, are the iso-frequency contours of the helicoid surface states at frequency $\omega_{0}$ labeled in (d), (e), (f). The glide planes are fill in gray and the $G_{i} \mathcal{T}$ protected line degeneracies are shown in dashed gray lines. (g), (h), (i) illustrates the gapless surface dispersions, around the projected monopoles, along a circular path of $\bar{k}_{\theta}$ in the surface BZ. The $Z$ monopoles can be Weyl points, nodal lines or surfaces. The $Z_{2}$ monpoles can be Dirac points, $Z_{2}$ nodal lines or Weyl dipoles.

example) cannot protect any line degeneracies on the surface, disallowing the gapless connectivity between two helicoids of opposite chiralities. In contrast, the $Z_{2}$ invariant of our acoustic Dirac point is protected by $G_{i} \mathcal{T}$ [27, leading to the nontrivial band topology not only in the bulk but also on the surface.

In Fig. 2(d), we calculate the non-Abelian Berry phase 34] of the lower two bands (5th and 6th) on a sphere enclosing the Dirac point. The gapless spectra indicate the nontrivial monopole charge of $Z_{2}=1$. Since this $Z_{2}$ charge can be protected by either one of the three $G_{i} \mathcal{T}$ [27, we can break the other two or one $G_{i} \mathcal{T}$ to get the $Z_{2}$ nodal ring [35-39] and Weyl dipoles [27, 31. These symmetry-breaking cases are illustrated in Fig. 2 (e), (f), (g)and discussed in detail in the Supplementary Materials.

Quad-helicoid and Jacobi elliptic function We project the two Dirac points onto the (001) surface, corresponding to the case in Fig. 1 (c), (f), (i). The plane group of this surface is $p 2 g g$, on which the two degenerate lines due to $G_{x} \mathcal{T}$ and $G_{y} \mathcal{T}$ are presented. These two line degeneracies, outlining the whole boundaries of the surface BZ, protect all the crossings among the four helicoid surface sheets. The iso-frequency contour, in Fig. 1.(c), are four branches originating from the projected Dirac points. The four branches are connected across the zone boundary forming two non-contractable loops.

If we parametrize the $2 \mathrm{D}$ surface $\mathrm{BZ}$ as a complex plane $\left(z \propto k_{x}+i k_{y}\right)$ [27, the helicoid surface bands can be expressed as (are topologically equivalent to) the double-periodic elliptic functions [40, 41]. The four helicoids in Fig. 1(f) are plotted using the Jacobi elliptic functions $\operatorname{cn}\left(z, \frac{1}{2}\right)$. The Jacobi function has two zeros and two poles in one period, each locating at the center of each quadrant. These four singularities represent two Weyl dipoles and all project to the same point in the surface BZ. So we construct the quad-helicoid surfaces by stacking the four quadrants of the Jacobi func- 
(a) BCC cubic cell

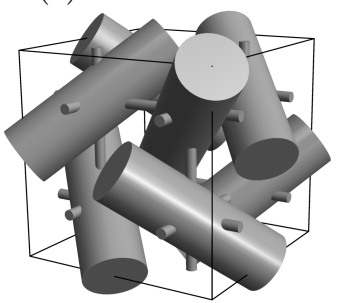

(d) Berry phase

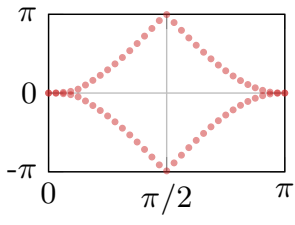

(e) $Z_{2}$ Dirac point (No. 206)

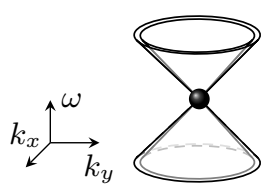

(b) Brillouin zone

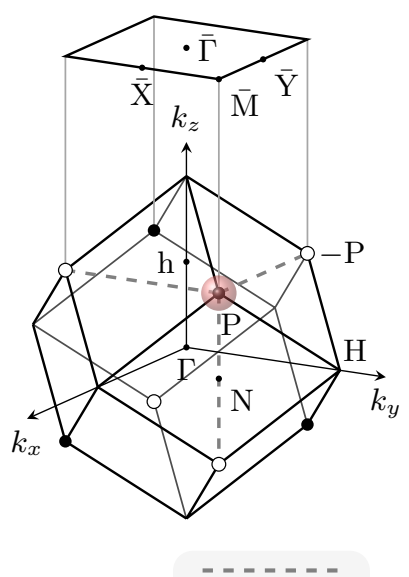

Line degeneracy

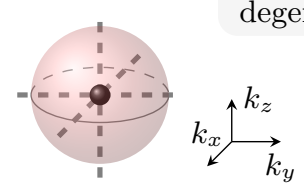

(c) Acoustic bulk bands and density of states

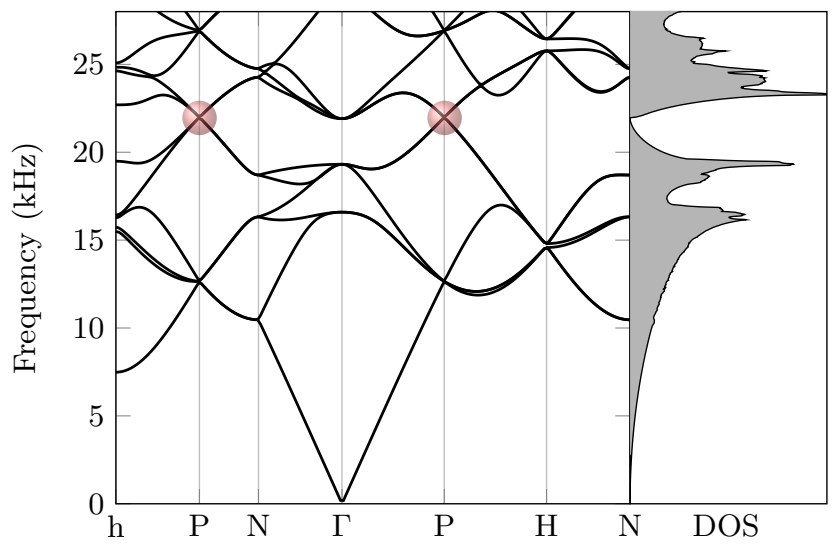

(f) $Z_{2}$ nodal ring (No. 015)

(g) $Z_{2}$ Weyl dipole (No. 045)

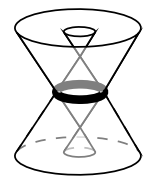

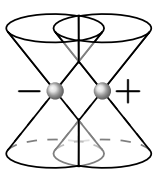

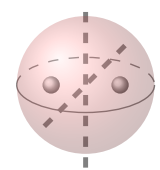

FIG. 2. Ideal acoustic Dirac points with $Z_{2}$ charges. (a) One cubic cell of the acoustic crystal of BCC lattice. (b) BCC BZ and the [001] surface BZ. The two $\pm P$ points project to the same point $\bar{M}$ in the surface BZ. (c) Acoustic bulk band structure with the Dirac points crossing between the 5-6th and 7-8th bands at the $P$ point. The DOS vanished at the frequency of ideal Dirac points. (d) Calculated non-Abelian Berry phases on the surface of the red sphere enclosing the Dirac point. Over the polar angle, the nontrivial winding of Berry phases implies topological invariant of $Z_{2}=1$. (e, f, g) Three types of four-bands nodal structures that carry $Z_{2}$ monopole charges. The dashed gray lines are the line degeneracies due to $G_{i} \mathcal{T}$, and the red spherical surfaces enclose the $Z_{2}$ monopoles.

tion and align the central singularities. The mapping of one quadrant is $\omega\left(k_{x}, k_{y}\right) \sim \operatorname{Im}\left(\log \left[\operatorname{cn}\left(z_{i}\left(k_{x}, k_{y}\right), \frac{1}{2}\right)\right]\right)$, where $z_{1}\left(k_{x}, k_{y}\right)=\frac{\mathrm{K}\left(\frac{1}{2}\right)}{\pi}\left(\frac{k_{x}-k_{y}}{2}+i \frac{k_{x}+k_{y}}{2}\right)$ and $\mathrm{K}$ is the complete elliptic integrals of the first kind. The rest three quadrants are obtained by the translations of $z_{2}\left(k_{x}, k_{y}\right)=z_{1}\left(k_{x}+2 \pi, k_{y}\right), z_{3}\left(k_{x}, k_{y}\right)=z_{1}\left(k_{x}, k_{y}+2 \pi\right)$, and $z_{4}\left(k_{x}, k_{y}\right)=z_{1}\left(k_{x}+2 \pi, k_{y}+2 \pi\right)$.

Experiments A photograph of the Dirac acoustic crystal is shown in Fig. 3(a), 3D-printed by the stereo lithography method using photocurable resin. The lattice constant is $a=20 \mathrm{~mm}$ and the fabrication error is $\pm 0.1 \mathrm{~mm}$. The total size of the sample is $413.0 \mathrm{~mm} \times 413.0 \mathrm{~mm} \times 222.8 \mathrm{~mm}$ containing $20 \times 20 \times 11 \mathrm{cu}-$ bic cells.

The surface states are measured through the Fouriertransformed field scan (FTFS). Similar approaches have been used to study other topological acoustic crystals 42 46. A pressure-field microphone (diameter of $3.5 \mathrm{~mm}, \mathrm{~B} \& \mathrm{~K}-4138-\mathrm{A}-015)$ is used as the scanning probe (receiver). The microphone is embedded in an aluminum alloy plate which works as a hard wall boundary on the top surface of the sample, as show in Fig. 3(a). The acoustic source is a broadband earphone, having frequency response up to $40 \mathrm{kHz}$ and a diameter of $5.5 \mathrm{~mm}$, fixed at the corner of the sample close to the plate. The amplitude and phase of the pressure field are collected by the data acquisition module B\&K-3160-A-042. A broadband signal is generated from the module and split into two channels, one to driven the earphone and the other as a time reference for the receiver. The frequency spectrum is averaged 100 times for each point scan and is normalized by the signal from the source.

The field scan is performed by moving the sample stage in three directions. During the collection of each data point, the sample is pressed towards the top plate to ensure the absence of air gaps. The scanning step is $5 \mathrm{~mm}$ and the scanning range is $400 \mathrm{~mm}$ in both $x$ and $y$ directions. Through $2 \mathrm{D}$ Fourier transforms, we obtain the spectral weight of the surface states in the momentum range of $(-2,2) \frac{2 \pi}{a}$. Similar to the processing technique used in Ref. [47, we patch the data of spatial scans to double the momentum resolution in the reciprocal space. In the $x$ direction, we stitch two scanning fields of equivalent source positions. In the $y$ direction, we rotate the data due to the $C_{2}$ symmetry on the surface.

The FTFS results are shown in three plots in Fig. 3 (d) (f) (h). The corresponding numerical results of the local density of states (LDOS) 48, 49 at the measurement interface are shown in Fig. 3 (c) (e) (g) respectively. The detailed algorithm of the surface LDOS will 
(a) Experiment setup

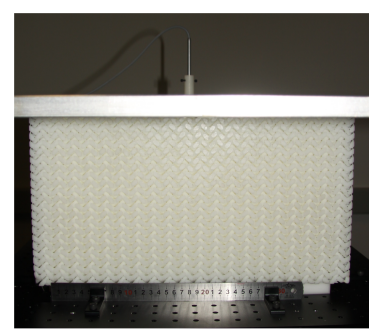

(b) Sample surface

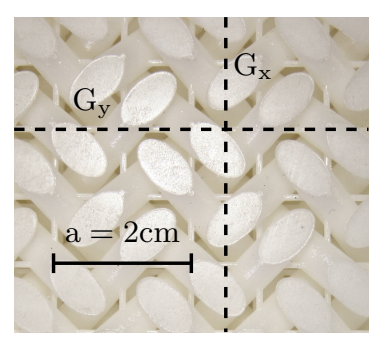

(g) Surface LDOS in circular route

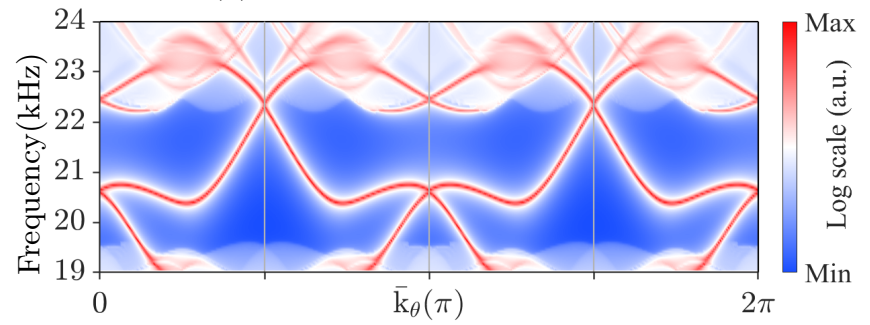

(c) Calculation of LDOS on the surface

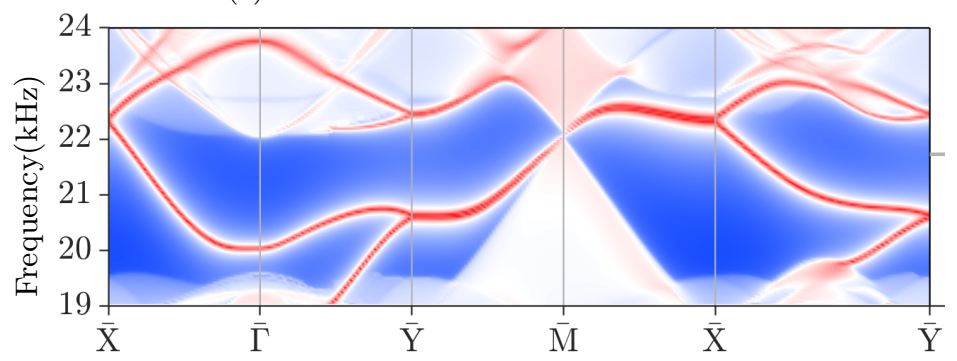

(d) Experimental result of surface states

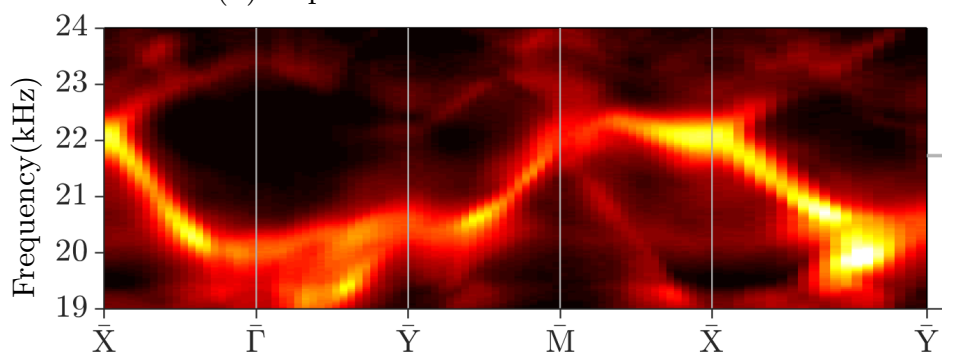

(e) Iso-frequency contour

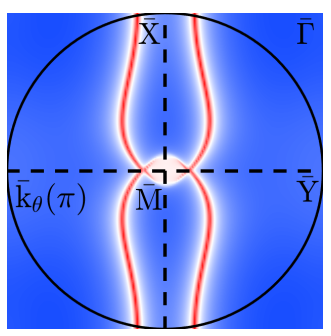

(f) Measured contour

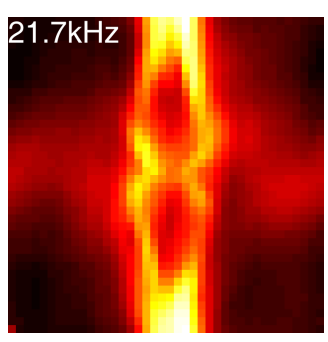

(h) Experimental surface states in circular route

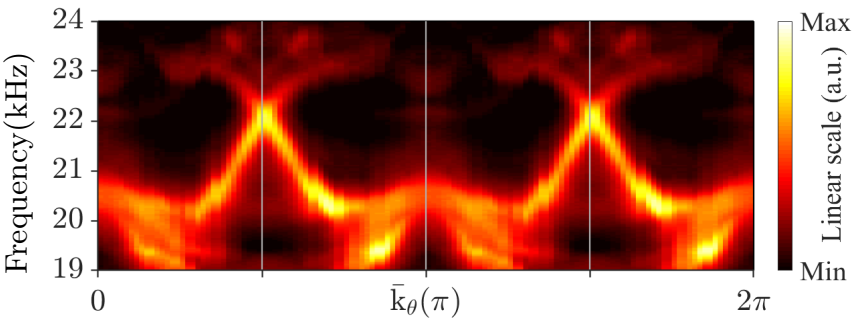

FIG. 3. Experiment observation of the quad-helicoid surface states by FTFS. (a) Photograph of the 3D-printed sample on the measurement setup. (b) Photograph of the sample surface. (c), (d) Numerical and experimental results of the surface states on the high-symmetry momentum lines. (e), (f) Numerical and experimental iso-frequency contour of the quad-helicoid surfaces showing four branches at $21.7 \mathrm{kHz}$. (g), (h) Numerical and experimental results of the surface states in a circular route around the projected bulk Dirac points.

be presented in a separate paper. The agreement between experiments and numerics are visually obviously for the gapless surface dispersions.

Discussion We experimentally observed the first example of topological surface states associated with the 3D Dirac points. The line-degeneracy due to glide and $\mathcal{T}$ symmetry is the key for stabilizing the gapless connection of helicoid sheets of opposite chiralities. Similarly, the other nonsymmorphic symmetry (screw rotation) and $\mathcal{T}$ could also protect such line degeneracies when a domain wall is constructed to preserve the screw axis on the surface. It will also be interesting to explore the material realization of the double-helicoid surface states shown in Fig. 1(e), as well as helicoid surface states of Dirac semimetals [27.

This work establishes an ideal 3D Dirac material for consequent studies. For example, symmetry breakings of Dirac points can generate a variety of topological phenomena, such as the charged $\left(Z\right.$ or $Z_{2}$ ) nodal lines, nodal surfaces [50, Weyl dipoles as well as a gapped bulk state supporting gapless surface 28] or chiral hinge modes [51, 52].

Acknowledgements We thank Timothy Hsieh and Liang $\mathrm{Fu}$ for the previous discussions on the $k \cdot p$ model. We are supported by the National key R\&D Program of China under Grant No. 2017YFA0303800, 2016YFA0302400, 2016YFA0300600, by NSFC under Project No. 11974415, 11721404, 11674370, and by Chinese Academy of Sciences under grant number XXH13506-202.

* linglu@iphy.ac.cn

[1] N. P. Armitage, E. J. Mele, and Ashvin Vishwanath. Weyl and dirac semimetals in three-dimensional solids. Reviews of Modern Physics, 90(1):15001, 2018.

[2] ZK Liu, Bo Zhou, Yong Zhang, ZJ Wang, HM Weng, Dharmalingam Prabhakaran, S-K Mo, ZX Shen, Zhong Fang, Xi Dai, et al. Discovery of a threedimensional topological dirac semimetal, na3bi. Science, 
343(6173):864-867, 2014.

[3] Madhab Neupane, Su Yang Xu, Raman Sankar, Nasser Alidoust, Guang Bian, Chang Liu, Ilya Belopolski, Tay Rong Chang, Horng Tay Jeng, Hsin Lin, Arun Bansil, Fangcheng Chou, and M. Zahid Hasan. Observation of a three-dimensional topological dirac semimetal phase in high-mobility cd3as2. Nature Communications, 5(1):3786, 2014.

[4] Zk Liu, J Jiang, B Zhou, Zj Wang, Y Zhang, Hm Weng, D Prabhakaran, Sk Mo, H Peng, $\mathrm{P}$ Dudin, T Kim, M Hoesch, Z Fang, X Dai, Zx Shen, Dl Feng, Z Hussain, and Yl Chen. A stable three-dimensional topological dirac semimetal cd3as2. Nature Materials, 13(7):677681, 2014.

[5] Borisenko S, Gibson Q, Evtushinsky D, Zabolotnyy, Bchner B, and Cava Rj. Experimental realization of a threedimensional dirac semimetal. Physical Review Letters, 113(2):27603, 2014.

[6] Su Yang Xu, Chang Liu, Satya K. Kushwaha, Raman Sankar, Jason W. Krizan, Ilya Belopolski, Madhab Neupane, Guang Bian, Nasser Alidoust, Tay Rong Chang, Horng Tay Jeng, Cheng Yi Huang, Wei Feng Tsai, Hsin Lin, Pavel P. Shibayev, Fang Cheng Chou, Robert J. Cava, and M. Zahid Hasan. Observation of fermi arc surface states in a topological metal. Science, 347(6219):294-298, 2015.

[7] Hemian Yi, Zhijun Wang, Chaoyu Chen, Youguo Shi, Ya Feng, Aiji Liang, Zhuojin Xie, Shaolong He, Junfeng He, Yingying Peng, Xu Liu, Yan Liu, Lin Zhao, Guodong Liu, Xiaoli Dong, Jun Zhang, M. Nakatake, M. Arita, K. Shimada, H. Namatame, M. Taniguchi, Zuyan Xu, Chuangtian Chen, Xi Dai, Zhong Fang, and X. J. Zhou. Evidence of topological surface state in three-dimensional dirac semimetal cd3as2. Scientific Reports, 4(1):61066106, 2015.

[8] Weiliang Yao, Chenyuan Li, Lichen Wang, Shangjie Xue, Yang Dan, Kazuki Iida, Kazuya Kamazawa, Kangkang Li, Chen Fang, and Yuan Li. Topological spin excitations in a three-dimensional antiferromagnet. Nature Physics, 14(10):1, 2018.

[9] Song Bao, Jinghui Wang, Wei Wang, Zhengwei Cai, Shichao Li, Zhen Ma, Di Wang, Kejing Ran, Zhao-Yang Dong, D. L. Abernathy, Shun-Li Yu, Xiangang Wan, Jian-Xin Li, and Jinsheng Wen. Discovery of coexisting dirac and triply degenerate magnons in a threedimensional antiferromagnet. Nature Communications, 9(1):2591, 2018.

[10] Qinghua Guo, Oubo You, Biao Yang, James B. Sellman, Edward Blythe, Hongchao Liu, Yuanjiang Xiang, Jensen Li, Dianyuan Fan, Jing Chen, C. T. Chan, and Shuang Zhang. Observation of three-dimensional photonic dirac points and spin-polarized surface arcs. Physical Review Letters, 122(20), 2019.

[11] Steve M Young, Saad Zaheer, Jeffrey CY Teo, Charles L Kane, Eugene J Mele, and Andrew M Rappe. Dirac semimetal in three dimensions. Physical review letters, 108(14):140405, 2012.

[12] Zhijun Wang, Yan Sun, Xingqiu Chen, Cesare Franchini, Gang Xu, Hongming Weng, Xi Dai, and Zhong Fang. Dirac semimetal and topological phase transitions in a(3)bi ( $\mathrm{a}=\mathrm{na}, \mathrm{k}, \mathrm{rb})$. Physical Review B, 85(19), 2012.

[13] Zhijun Wang, Hongming Weng, Quansheng Wu, Xi Dai, and Zhong Fang. Three-dimensional dirac semimetal and quantum transport in cd3as2. Physical Review B, 88(12), 2013.

[14] Julia A. Steinberg, Steve M. Young, Saad Zaheer, C. L. Kane, E. J. Mele, and Andrew M. Rappe. Bulk dirac points in distorted spinels. Physical Review Letters, 112(3):36403, 2014.

[15] HaiXiao Wang, Lin Xu, HuanYang Chen, and Jian-Hua Jiang. Three-dimensional photonic dirac points stabilized by point group symmetry. Physical Review B, 93(23):235155, 2016.

[16] Peizhe Tang, Quan Zhou, Gang Xu, and ShouCheng Zhang. Dirac fermions in an antiferromagnetic semimetal. Nature Physics, 12(12):1100-1104, 2016.

[17] Gang Li, Binghai Yan, Zhijun Wang, and Karsten Held. Topological dirac semimetal phase in pd and pt oxides. Physical Review B, 95(3):1-7, 2017.

[18] Alexey Slobozhanyuk, S. Hossein Mousavi, Xiang Ni, Daria Smirnova, Yuri S. Kivshar, and Alexander B. Khanikaev. Three-dimensional all-dielectric photonic topological insulator. Nature Photonics, 11(2):130-136, 2017.

[19] Qinghua Guo, Biao Yang, Lingbo Xia, Wenlong Gao, Hongchao Liu, Jing Chen, Yuanjiang Xiang, and Shuang Zhang. Three dimensional photonic dirac points in metamaterials. Physical Review Letters, 119(21), 2017.

[20] Hai-Xiao Wang, Yige Chen, Zhi Hong Hang, Hae-Young Kee, and Jian-Hua Jiang. Type-ii dirac photons. npj Quantum Materials, 2(1):54, 2017.

[21] Congcong Le, Xianxin Wu, Shengshan Qin, Yinxiang Li, Ronny Thomale, Fuchun Zhang, and Jiangping Hu. Dirac semimetal in -cui without surface fermi arcs. Proceedings of the National Academy of Sciences of the United States of America, 115(33):201803599, 2018.

[22] Peng Zhang, Zhijun Wang, Xianxin Wu, Koichiro Yaji, Yukiaki Ishida, Yoshimitsu Kohama, Guangyang Dai, Yue Sun, Cedric Bareille, Kenta Kuroda, Takeshi Kondo, Kozo Okazaki, Koichi Kindo, Xiancheng Wang, Changqing Jin, Jiangping Hu, Ronny Thomale, Kazuki Sumida, Shilong Wu, Koji Miyamoto, Taichi Okuda, Hong Ding, G.D. Gu, Tsuyoshi Tamegai, Takuto Kawakami, Masatoshi Sato, and Shik Shin. Multiple topological states in iron-based superconductors. Nature Physics, 15(1):41-47, 2019.

[23] Yihao Yang, Zhen Gao, Haoran Xue, Li Zhang, Mengjia He, Zhaoju Yang, Ranjan Singh, Yidong Chong, Baile Zhang, and Hongsheng Chen. Realization of a threedimensional photonic topological insulator. Nature, 565(7741):622-626, 2019.

[24] Mehdi Kargarian, Mohit Randeria, and Yuan-Ming Lu. Are the surface fermi arcs in dirac semimetals topologically protected. Proceedings of the National Academy of Sciences of the United States of America, 113(31):86488652, 2016.

[25] Mehdi Kargarian, Yuan-Ming Lu, and Mohit Randeria. Deformation and stability of surface states in dirac semimetals. Physical Review B, 97(16):165129, 2018.

[26] Yun Wu, Na Hyun Jo, Lin-Lin Wang, Connor A. Schmidt, Kathryn M. Neilson, Benjamin Schrunk, Przemyslaw Swatek, Andrew Eaton, S. L. Bud'ko, P. C. Canfield, and Adam Kaminski. Fragility of fermi arcs in dirac semimetals. Physical Review B, 99(16), 2019.

[27] Chen Fang, Ling Lu, Junwei Liu, and Liang Fu. Topological semimetals with helicoid surface states. Nature Physics, 12(10):936, 2016. 
[28] Ling Lu, Chen Fang, Liang Fu, Steven G Johnson, John D Joannopoulos, and Marin Soljačić. Symmetryprotected topological photonic crystal in three dimensions. Nature Physics, 12(4):337, 2016.

[29] Ling Lu, Chen Fang, Timothy H. Hsieh, Liang Fu, Steven G. Johnson, John D. Joannopoulos, and Marin Soljai. Generalized three-dimensional dirac points and $\mathrm{z}$ 2 gapless surface states in a topological photonic crystal. In 2015 Conference on Lasers and Electro-Optics (CLEO), pages 1-2, 2015.

[30] Boyuan Liu, J D Joannopoulos, Steven G Johnson, and Ling Lu. Generalized gilatraubenheimer method for density-of-states calculation in photonic crystals. Journal of Optics, 20(4):44005, 2018.

[31] Takahiro Morimoto and Akira Furusaki. Weyl and dirac semimetals with z2 topological charge. Physical Review B, 89(23), 2014.

[32] Bohm-Jung Yang and Naoto Nagaosa. Classification of stable three-dimensional dirac semimetals with nontrivial topology. Nature Communications, 5(1):4898, 2014.

[33] Bohm-Jung Yang, Takahiro Morimoto, and Akira Furusaki. Topological charges of three-dimensional dirac semimetals with rotation symmetry. Physical Review B, 92(16), 2015.

[34] Rui Yu, Xiao Liang Qi, Andrei Bernevig, Zhong Fang, and Xi Dai. Equivalent expression of $\mathrm{z}(2)$ topological invariant for band insulators using the non-abelian berry connection. Physical Review B, 84(7):75119, 2011.

[35] Chen Fang, Yige Chen, Hae-Young Kee, and Liang Fu. Topological nodal line semimetals with and without spinorbital coupling. Physical Review B, 92(8):81201, 2015.

[36] Kangkang Li, Chenyuan Li, Jiangping Hu, Yuan Li, and Chen Fang. Dirac and nodal line magnons in threedimensional antiferromagnets. Physical Review Letters, 119(24), 2017.

[37] Tom Bzduek and Manfred Sigrist. Robust doubly charged nodal lines and nodal surfaces in centrosymmetric systems. Physical Review B, 96(15):155105, 2017.

[38] Zhida Song, Tiantian Zhang, and Chen Fang. Diagnosis for nonmagnetic topological semimetals in the absence of spin-orbital coupling. Physical Review X, 8(3), 2018.

[39] Junyeong Ahn, Dongwook Kim, Youngkuk Kim, and Bohm-Jung Yang. Band topology and linking structure of nodal line semimetals with z2 monopole charges. Physical Review Letters, 121(10), 2018.

[40] Tiantian Zhang, Zhida Song, A Alexandradinata, Hongming Weng, Chen Fang, Ling Lu, and Zhong Fang. Double-weyl phonons in transition-metal monosilicides. Physical review letters, 120(1):016401, 2018.

[41] Biao Yang, Qinghua Guo, Ben Tremain, Rongjuan Liu, Lauren E Barr, Qinghui Yan, Wenlong Gao, Hongchao Liu, Yuanjiang Xiang, Jing Chen, et al. Ideal weyl points and helicoid surface states in artificial photonic crystal structures. Science, 359(6379):1013-1016, 2018.

[42] Feng Li, Xueqin Huang, Jiuyang Lu, Jiahong Ma, and Zhengyou Liu. Weyl points and fermi arcs in a chiral phononic crystal. Nature Physics, 14(1):30-34, 2018.

[43] Hao Ge, Xu Ni, Yuan Tian, Samit Kumar Gupta, MingHui Lu, Xin Lin, Wei-Dong Huang, C. T. Chan, and Yan-Feng Chen. Experimental observation of acoustic weyl points and topological surface states. Physical review applied, 10(1), 2018.

[44] Yihao Yang, Hong xiang Sun, Jian ping Xia, Haoran Xue, Zhen Gao, Yong Ge, Ding Jia, Shou qi Yuan, Yidong
Chong, and Baile Zhang. Topological triply degenerate point with double fermi arcs. Nature Physics, page 1, 2019.

[45] Valerio Peri, Marc Serra-Garcia, Roni Ilan, and Sebastian D. Huber. Axial-field-induced chiral channels in an acoustic weyl system. Nature Physics, 15(4):357-361, 2019.

[46] Boyang Xie, Hui Liu, Hua Cheng, Zhengyou Liu, Shuqi Chen, and Jianguo Tian. Experimental realization of type-ii weyl points and fermi arcs in phononic crystal. Physical Review Letters, 122(10), 2019.

[47] Qinghui Yan, Rongjuan Liu, Zhongbo Yan, Boyuan Liu, Hongsheng Chen, Zhong Wang, and Ling Lu. Experimental discovery of nodal chains. Nature Physics, 14(5):461464, 2018.

[48] M P Lopez Sancho, J M Lopez Sancho, J M L Sancho, and J Rubio. Highly convergent schemes for the calculation of bulk and surface green functions. Journal of Physics F: Metal Physics, 15(4):851-858, apr 1985.

[49] QuanSheng Wu, ShengNan Zhang, Hai-Feng Song, Matthias Troyer, and Alexey A. Soluyanov. Wanniertools : An open-source software package for novel topological materials. Computer Physics Communications, 224:405 - 416, 2018.

[50] Meng Xiao and Shanhui Fan. Topologically charged nodal surface. Preprint at arXiv: 1709.02363, 2017.

[51] Changming Yue, Yuanfeng Xu, Zhida Song, Hongming Weng, Yuan-Ming Lu, Chen Fang, and Xi Dai. Symmetry-enforced chiral hinge states and surface quantum anomalous hall effect in the magnetic axion insulator bi2xsmxse3. Nature Physics, 15(6):577-581, 2019.

[52] Heejae Kim, Ken Shiozaki, and Shuichi Murakami. Glidesymmetric magnetic topological crystalline insulators with inversion symmetry. Phys. Rev. B, 100:165202, Oct 2019 . 


\title{
Discovering topological surface states of Dirac points: Supplementary material
}

\author{
Hengbin Cheng, ${ }^{1,2}$ Yixin Sha, ${ }^{3}$ Rongjuan Liu, ${ }^{1}$ Chen Fang, ${ }^{1}$ and Ling Lu ${ }^{1,4}$ \\ ${ }^{1}$ Institute of Physics, Chinese Academy of Sciences/Beijing National \\ Laboratory for Condensed Matter Physics, Beijing 100190, China \\ ${ }^{2}$ School of Physical Sciences, University of Chinese Academy of Sciences, Beijing 100049, China \\ ${ }^{3}$ School of Electronics Engineering and Computer Science, Peking University, Beijing 100871, China \\ ${ }^{4}$ Songshan Lake Materials Laboratory, Dongguan, Guangdong 523808, China.
}

\section{UNIT CELL DESIGN}

(a)No. 230 cubic cell

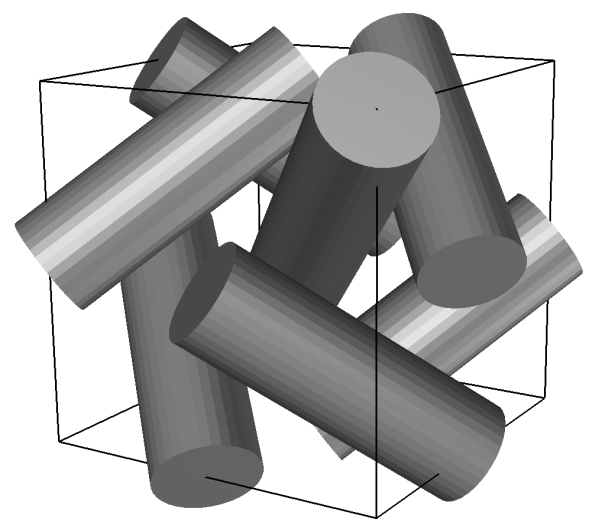

(c)No. 230 acoustic bulk bands and DOS

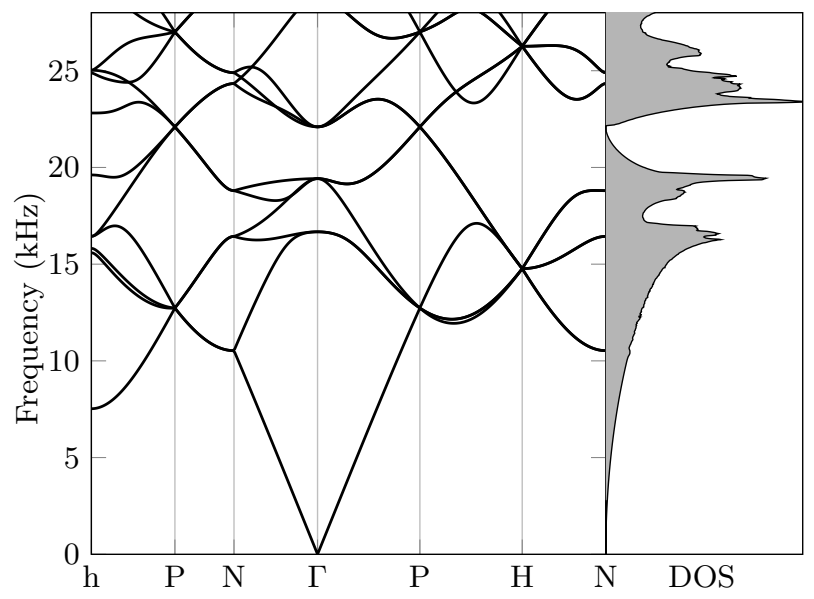

(b)No. 206 cubic cell

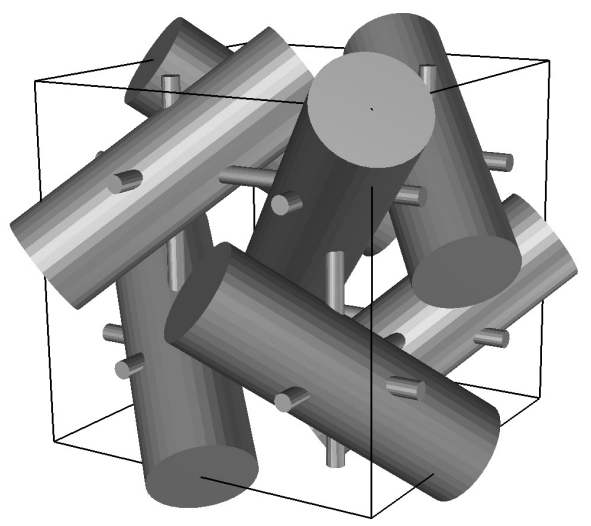

(d)No. 206 acoustic bulk bands and DOS

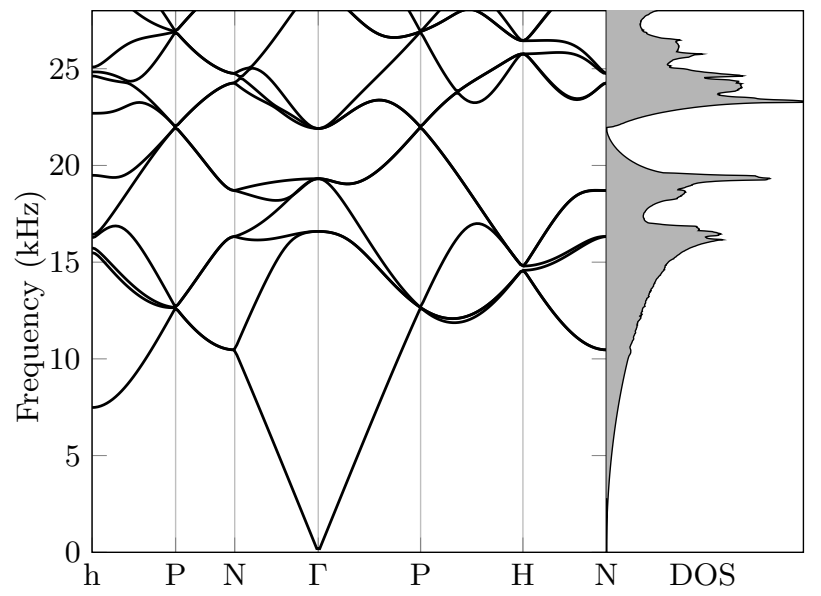

FIG. S1: Design of ideal acoustic Dirac point without and with the thin sticks. (a), (c) BPI acoustic crystal of space group No. 230. (b), (d) Thin-stick joined BPI acoustic crystal of space group No. 206.

For sample stability, we add the 12 thin sticks of radius $0.025 a$ ( $1 \mathrm{~mm}$ in experiment) to connect all the BPI rods. Fig. S1(a), (b) are the unit-cell structures without and with thin sticks, they belongs to space group No. 230 and No. 206 respectively. The thin sticks preserve the three glides that protect the ideal Dirac points. The corresponding acoustic bulk bands are plotted in Fig. S1(c), (d), which are almost identical for the frequency range of the Dirac points. 


\section{II. $\mathrm{k} \cdot \mathrm{p}$ MODEL}

The local dispersion and topology at $P$ point can be described by the effective Hamiltonian of $\boldsymbol{k} \cdot \boldsymbol{p}$ model, written as $H(\boldsymbol{k})=A \boldsymbol{k} \cdot \boldsymbol{p}$, considering the first non-zero order of $\boldsymbol{k}$. Here, we show the detailed derivation of the effective Hamiltonian of our ideal acoustic Dirac point in space group $I a \overline{3} d$ (No. 230) and $I a \overline{3}$ (No. 206).

Considering the space group $I a \overline{3} d$ (No. 230), we choose four independent symmetry operations (generators) of the little group at momentum point $P$. They are two-fold rotation symmetry $C_{2 z}=\left\{C_{2} \mid\left(0, \frac{1}{2}, 0\right)\right\}$ along [001] direction, two-fold rotation symmetry $C_{2 y}=\left\{C_{2} \mid\left(\frac{1}{2}, 0,0\right)\right\}$ along [010] direction, three-fold rotation symmetry $C_{3}$ along [111] direction and glide reflection symmetry $G=\left\{M \mid\left(-\frac{3}{4},-\frac{3}{4}, \frac{1}{4}\right)\right\}$ in [1110] plane. Here $G$ is different from the $G_{i}(i=x, y, z)$ glides in [100], [010], [001] planes. $P$ point has no $G_{i}(i=x, y, z)$ symmetries, but has $G_{i} \mathcal{T}$ that can be obtained by $G_{i} \mathcal{T}=C_{2 i} \cdot \mathcal{P} \mathcal{T}(i=x, y, z) . \mathcal{P} \mathcal{T}$ is the parity-time symmetry preserves in the whole BZ.

$P$ point has $\mathcal{P} \mathcal{T}$ but has neither $\mathcal{P}$ nor $\mathcal{T} . \mathcal{P} \mathcal{T}$ is an anti-unitary operator which can be written as $\mathcal{P} \mathcal{T}=U \cdot \mathcal{K}$, where $U$ is a unitary matrix and $\mathcal{K}$ is the complex conjugate. The little group of $P$ point has two conjugated $2 \mathrm{D}$ representations, forming a $4 \mathrm{D}$ representation under $\mathcal{P} \mathcal{T}$. We obtain the irreducible representation (Irrep) matrices of the selected generators from Bilbao crystal server, noted as Bilbao Rep. in Table II. After unitary transformations, we have the representation matrices in real basis (Real Rep.) and in Dirac basis (Dirac Rep.).

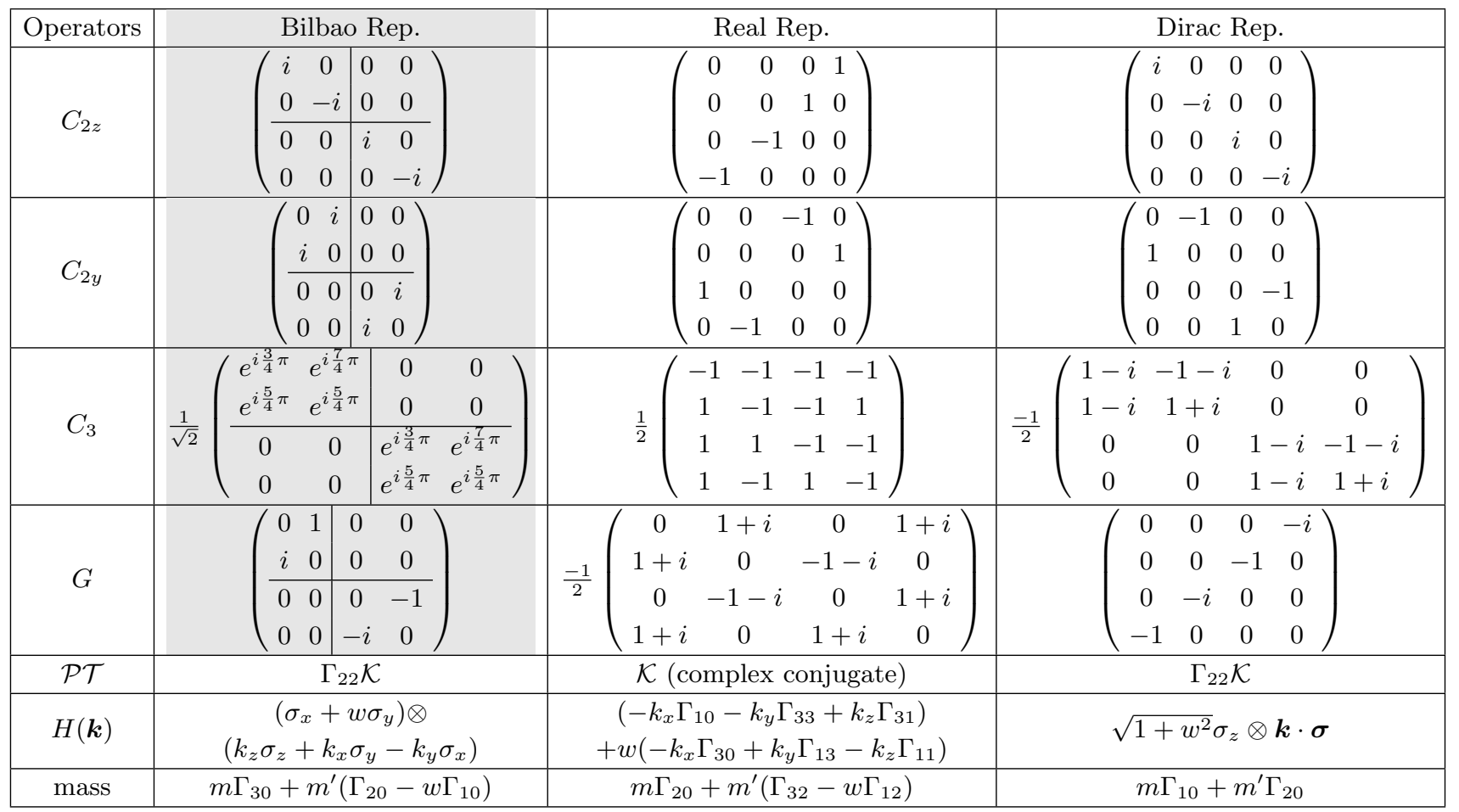

TABLE I: Representation matrices of selected generators of the little group at $P$ in space group No. 230. The four matrices in the gray cells are obtained from Bilbao website and the rest are worked out by commutation relations.

We need to find the representation matrix for $\mathcal{P} \mathcal{T}$ which is not given by Bilbao. We expand the $4 \mathrm{D}$ unitary $U$ matrix by the 16 Gamma matrices with complex coefficients and determine the coefficients by the commutation relations in Table II.

\begin{tabular}{c|c|c|c|c|c|c}
\hline & \multicolumn{2}{|c|}{$\mathcal{P} \mathcal{T}=U \cdot \mathcal{K}$} & $C_{2 z}$ & $C_{2 y}$ & $G$ & $C_{3}$ \\
\hline $\mathcal{P T}$ & $\mathcal{P} \mathcal{T}^{2}=1$ & $U U^{\dagger}=1$ & {$\left[C_{2 z}, \mathcal{P} \mathcal{T}\right]=0$} & {$\left[C_{2 y}, \mathcal{P} \mathcal{T}\right]=0$} & $G(\mathcal{P} \mathcal{T})=i(\mathcal{P T}) G$ & {$\left[C_{3}, \mathcal{P} \mathcal{T}\right]=0$} \\
\hline
\end{tabular}

TABLE II: Compatibility relations used to determine $\mathcal{P} \mathcal{T}$.

The sixteen Gamma matrices are $\Gamma_{i j}=\sigma_{i} \otimes \sigma_{j},(i, j=0,1,2,3)$, where $\sigma_{0}=\left(\begin{array}{cc}1 & 0 \\ 0 & 1\end{array}\right), \sigma_{1}=\left(\begin{array}{cc}0 & 1 \\ 1 & 0\end{array}\right), \sigma_{2}=\left(\begin{array}{cc}0 & -i \\ i & 0\end{array}\right), \sigma_{3}=$ $\left(\begin{array}{cc}1 & 0 \\ 0 & -1\end{array}\right)$, and $\otimes$ is the Kronecker product.

The Hamiltonian can be written as $H(\boldsymbol{k})=A\left(k_{x} p_{x}+k_{y} p_{y}+k_{z} p_{z}\right)$, where $k_{x}, k_{y}, k_{z}$ are three real variables (the origin is set at $P$ in below), and $p_{x}, p_{y}, p_{z}$ are three Hermitian matrices. To find $p_{x}, p_{y}, p_{z}$, we expand them as 
linear combinations of 16 Gamma matrices with real coefficients and determine the coefficient by the commutation relations in Table. III.

For any representation matrix $D(g)$ of symmetry operation $g$ (including $\mathcal{P} \mathcal{T}$ ) in the little group, we have

$$
D(g) H(\boldsymbol{k}) D^{-1}(g)=H(g \boldsymbol{k})
$$

that gives the following commutation $([]=0)$ and anti-commutation $(\{\}=0)$ relations, listed in Table. III.

\begin{tabular}{|c|c|c|c|}
\hline & $p_{z}$ & $p_{y}$ & $p_{x}$ \\
\hline $\mathcal{P} \mathcal{T}$ & {$\left[\mathcal{P} \mathcal{T}, p_{z}\right]=0$} & {$\left[\mathcal{P} \mathcal{T}, p_{y}\right]=0$} & {$\left[\mathcal{P} \mathcal{T}, p_{x}\right]=0$} \\
\hline$C_{2 z}$ & {$\left[C_{2 z}, p_{z}\right]=0$} & $\left\{C_{2 z}, p_{y}\right\}=0$ & $\left\{C_{2 z}, p_{x}\right\}=0$ \\
\hline$C_{2 y}$ & $\left\{C_{2 y}, p_{z}\right\}=0$ & {$\left[C_{2 y}, p_{y}\right]=0$} & $\left\{C_{2 y}, p_{x}\right\}=0$ \\
\hline$G$ & {$\left[G, p_{z}\right]=0$} & \multicolumn{2}{|c|}{$G p_{y}=p_{x} G$} \\
\hline$C_{3}$ & \multicolumn{2}{|c|}{$p_{y}=C_{3}^{-1} p_{z} C_{3}, p_{x}=C_{3} p_{z} C_{3}^{-1}$} \\
\hline
\end{tabular}

TABLE III: Compatibility relations used to determine $H(\boldsymbol{k})$.

By satisfying all the requirements in Table. III, for Bilbao Rep. we obtain $p_{z}=\Gamma_{13}+w \Gamma_{23},(w \in \mathbb{R})$. $p_{x}$ and $p_{y}$ are obtained from $p_{z}$ by $C_{3}$ symmetry. Ignore the constant term $A$, the effective Hamiltonian is given as

$$
H_{D}(\boldsymbol{k}) \sim\left(\sigma_{x}+w \sigma_{y}\right) \otimes\left(k_{z} \sigma_{z}+k_{x} \sigma_{y}-k_{y} \sigma_{x}\right)
$$

After an unitary transformation, we get the representation matrix under Dirac Rep.

$$
H_{D}(\boldsymbol{k}) \sim \sqrt{1+w^{2}}\left(\begin{array}{cc}
\boldsymbol{k} \cdot \boldsymbol{\sigma} & 0 \\
0 & -\boldsymbol{k} \cdot \boldsymbol{\sigma}
\end{array}\right)
$$

where $w \in \mathbb{R}$ is the velocity term. Note that the Dirac Hamiltonian we obtained above satisfies the exact massless Dirac equation in 3D. What listed in the last row of Table. I are the matrix of the Dirac mass terms, in corresponding basis with coefficients $m, m^{\prime} \in \mathbb{R}$.

For space group No. 206, the only missing generators is $G=\left\{M \mid\left(-\frac{3}{4},-\frac{3}{4}, \frac{1}{4}\right)\right\}$ in $[1 \overline{1} 0]$ plane, and the Dirac Hamiltonian under Bilbao Rep. is

$$
H_{D}(\boldsymbol{k}) \sim\left(\sigma_{x}+w \sigma_{y}+u \sigma_{z}\right) \otimes\left(k_{z} \sigma_{z}-k_{x} \sigma_{x}-k_{y} \sigma_{y}\right),(w, u \in \mathbb{R})
$$

. After an unitary transformation, we have the representation matrix under Dirac Rep.

$$
H_{D}(\boldsymbol{k}) \sim \sqrt{1+w^{2}+u^{2}}\left(\begin{array}{cc}
\boldsymbol{k} \cdot \boldsymbol{\sigma} & 0 \\
0 & -\boldsymbol{k} \cdot \boldsymbol{\sigma}
\end{array}\right)
$$




\section{DIRAC POINT UNDER SYMMETRY BREAKINGS}

Dirac point lies in the phase transition center of many topological band nodal structures. In Table. IV we list the possible four bands nodal structures that can be achieved from perturbing the Dirac point, and list the corresponding key symmetries that need to be $\operatorname{preserved}(\sqrt{ })$ or $\operatorname{broken}(\times)$.

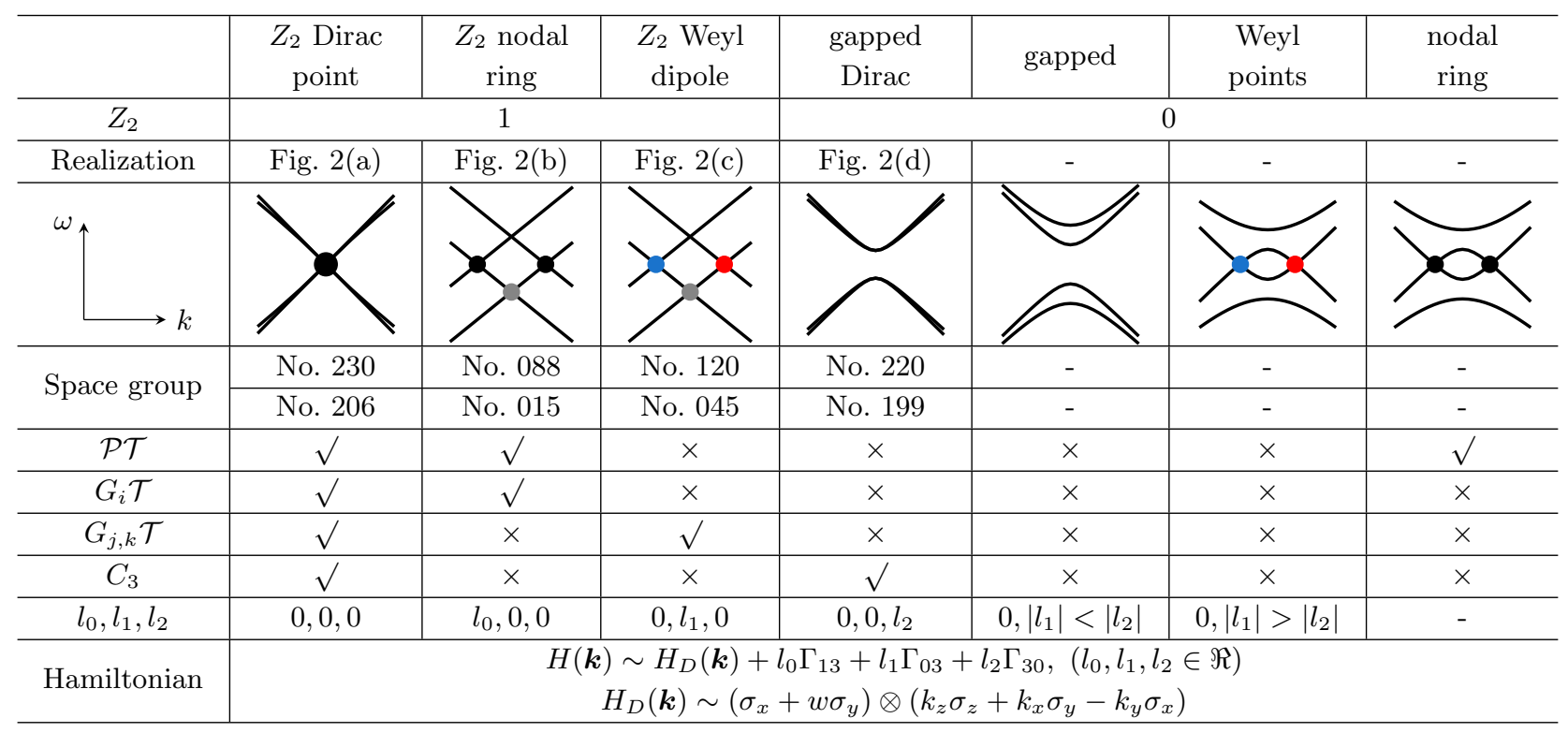

TABLE IV: Possible results of the Dirac point transition under symmetry breaking. 


\section{REALIZATION OF VARIOUS SYMMETRY BREAKINGS}

Unit cell

(a) No. 230

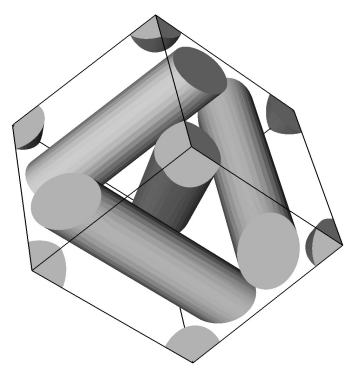

(b) No. 088

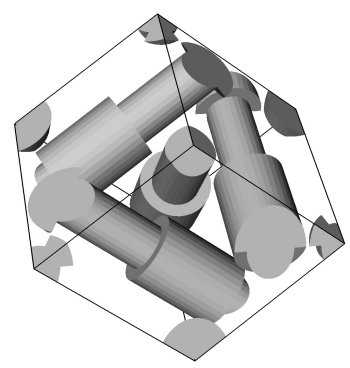

(c) No. 120

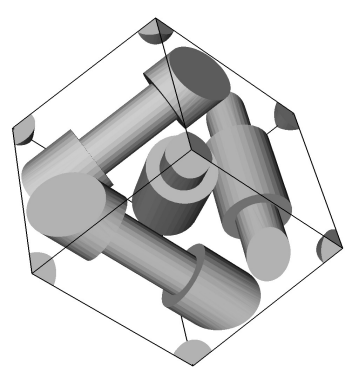

Acoustic bulk band structure

(d)

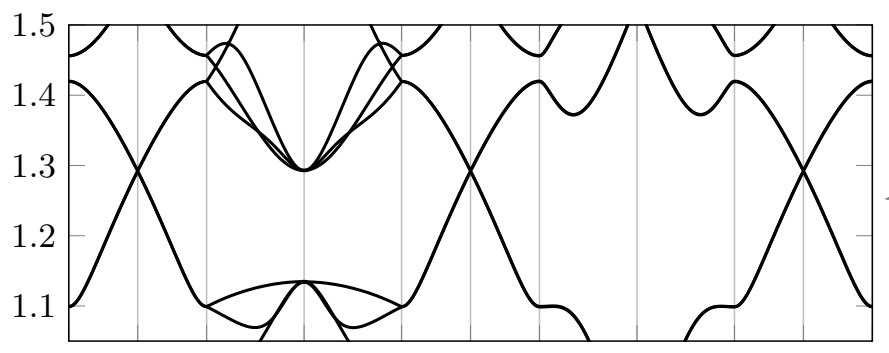

(e)

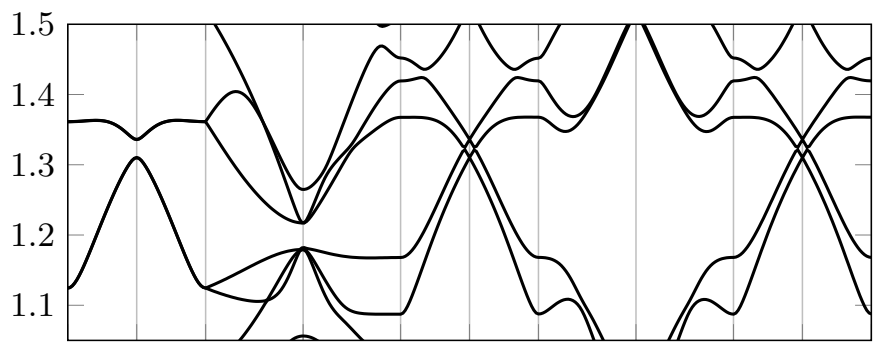

(f)

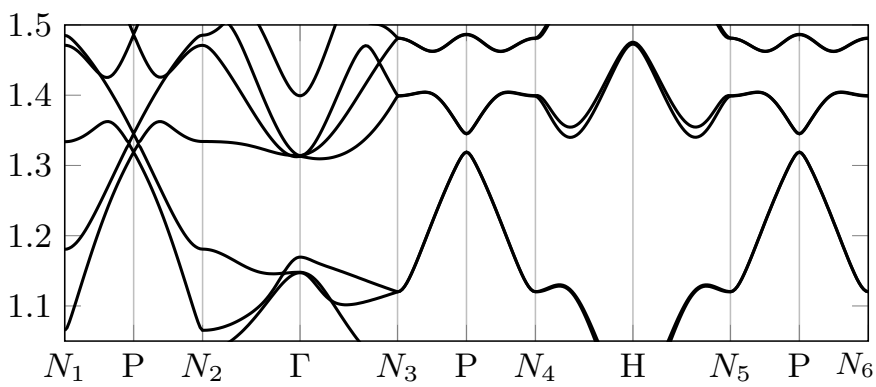

Degeneracies in BZ

(g) $Z_{2}$ Dirac points

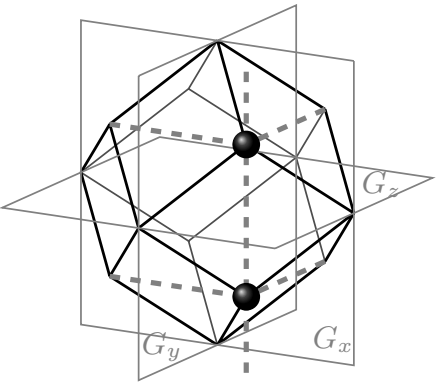

(h) $Z_{2}$ nodal rings

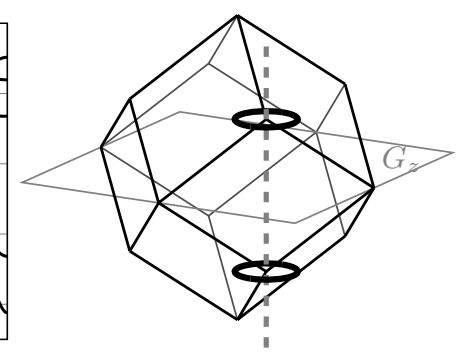

(i) $Z_{2}$ Weyl dipoles

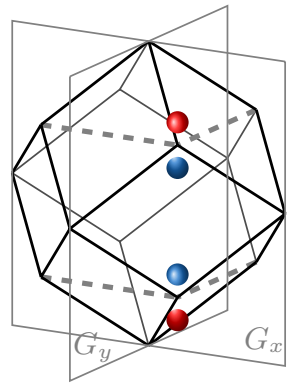

(j)

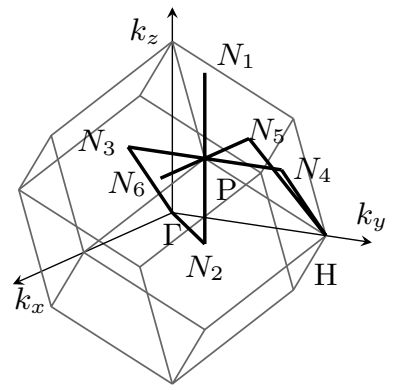

FIG. S2: Realization of the predicted symmetry breaking results. (a), (d), (g) Two Dirac points (black spheres) in the first BZ, locate at point $\pm P$. The dashed gray lines denote for the $G_{i} \mathcal{T}$ stabilized line degeneracies. (b), (e), (h) The Dirac points turn into $Z_{2}$ nodal rings (black circle). The $G_{z} \mathcal{T}$ stabilized degenerate lines, between lower or higher two bands, perpendicularly cross the rings and form nodal links within the four bands. (c),(f),(i) The Dirac points split into $Z_{2}$ Weyl dipoles (blue and red spheres for opposite chiralities). Since the two surface glides are not broken, the quad-helicoid surface states are still robust. Note that each one of $G_{x} \mathcal{T}, G_{y} \mathcal{T}$ is enough for keeping the $Z_{2}$ Weyl dipoles, but we need both to keep the quad-helicoid on 001 surface. 


\section{COMPARISON OF EIGENVALUE AND LDOS CALCULATIONS}

(a) Eigenvalue calculation of surface states

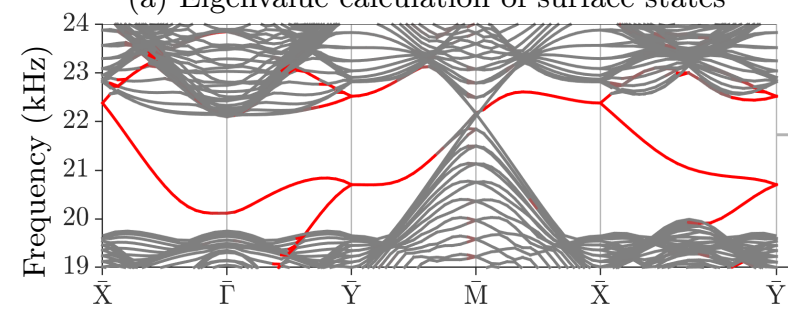

(c) Eigenvalue calculation of surface states

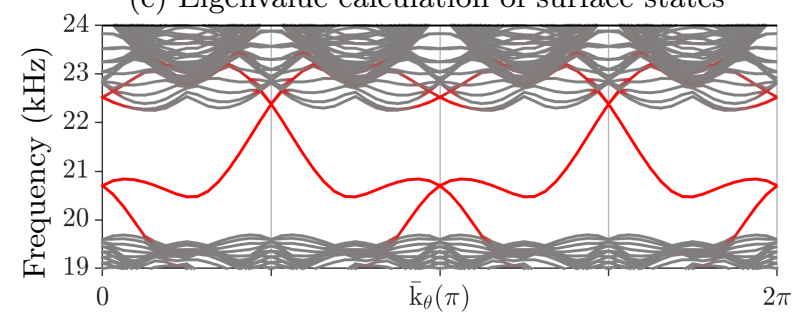

(b) LDOS calculation of surface states

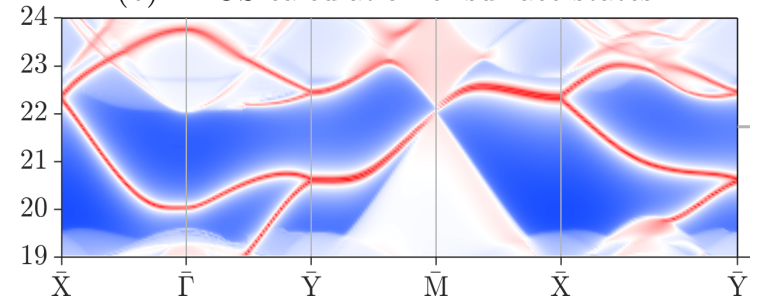

(d) LDOS calculation of surface states

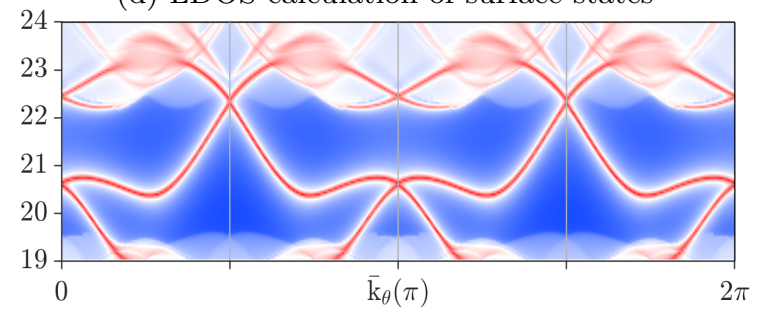

FIG. S3: Surface states in eigenvalue solutions and LDOS results. (a),(c) The surface states dispersion are obtained by solving the eigenvalue equation for a ten cubic cell stack with Floquet periodic boundary conditions in $\mathrm{x}$ and $\mathrm{y}$ directions and hard-wall boundaries on $\mathrm{z}$ and $\mathrm{-z}$ ends. The dispersion lines were colored in red if they have a large energy fraction in the first two cells on the surface. The results agree the local density of states (LDOS) results in (b), (d), respectively. 
VI. ADDITIONAL EXPERIMENTAL RESULTS

(a) Numerical result of surface states

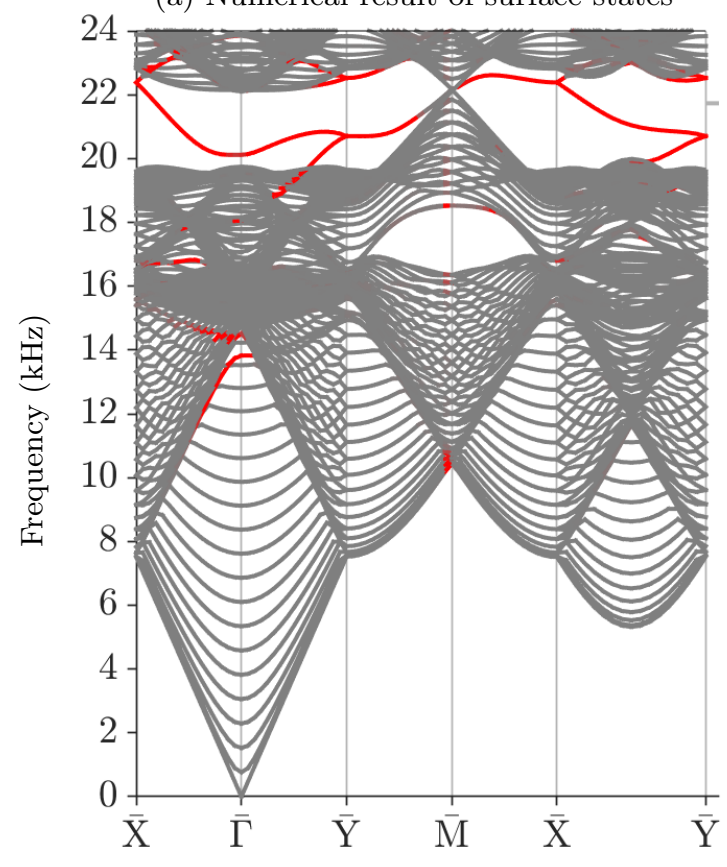

(c) Numerical result of surface states

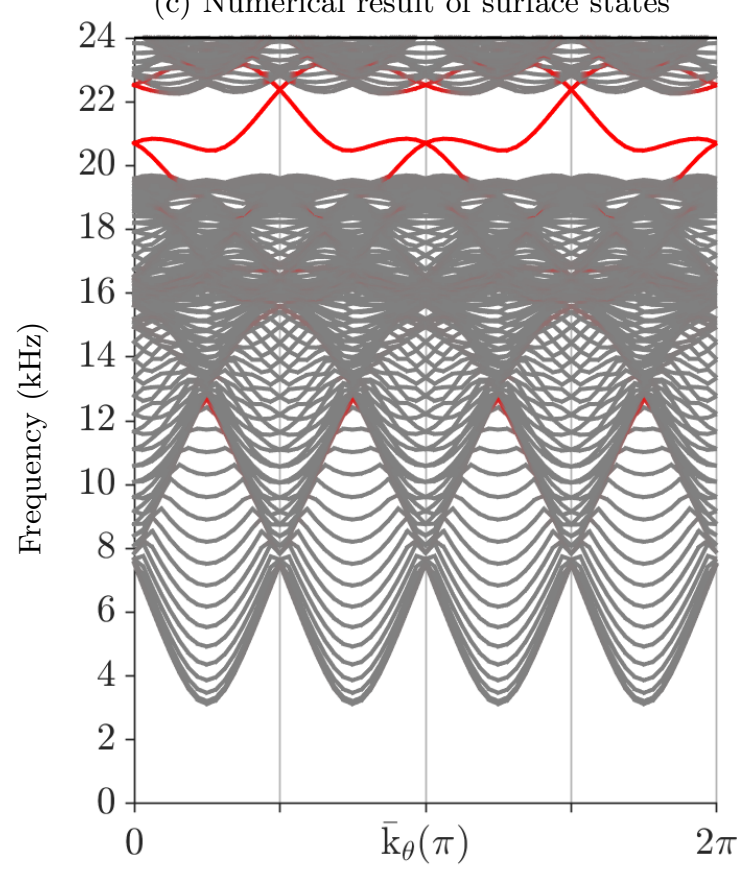

(b) Experimental result of surface states

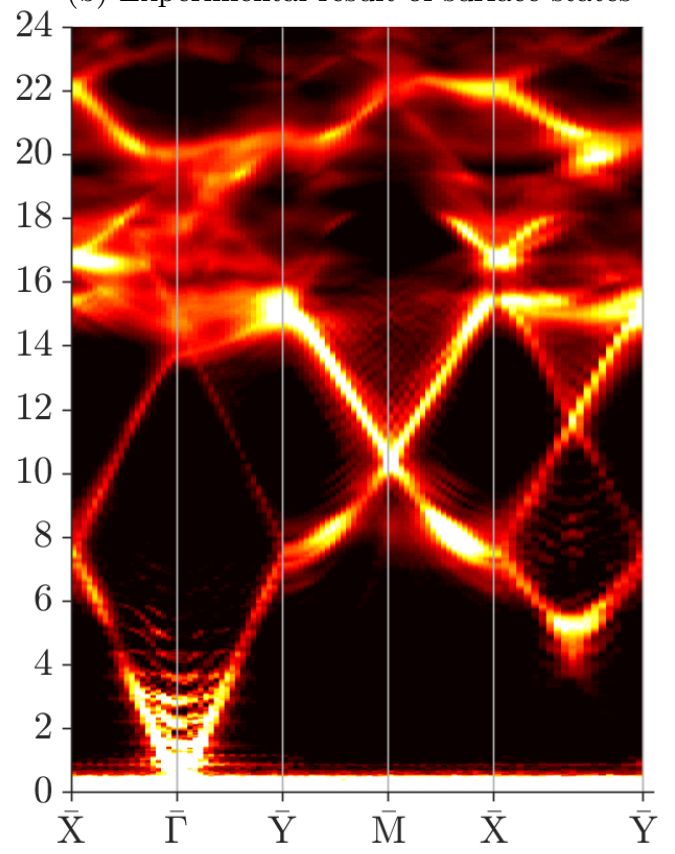

(d) Experimental result of surface states

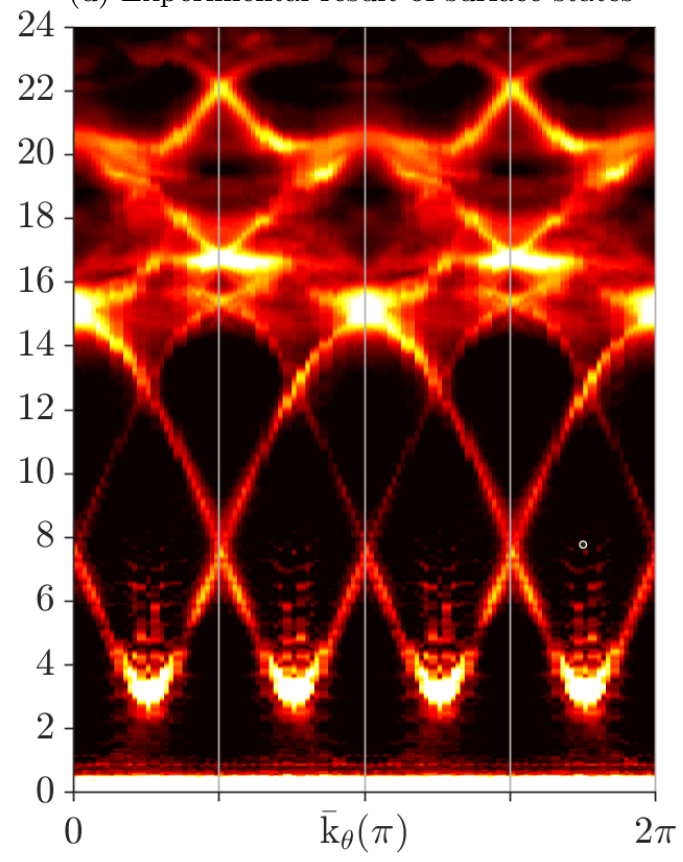

FIG. S4: Full frequency range data of the numerical and measured surface states. 
VII. MORE DETAILS ON EXPERIMENTAL MEASUREMENTS

\begin{tabular}{|c|c|c|c|}
\hline & $\begin{array}{c}\text { Mass density } \\
\rho\left(\mathrm{kg} / \mathrm{m}^{3}\right)\end{array}$ & $\begin{array}{c}\text { Longitudinal sound velocity } \\
v_{L}(\mathrm{~m} / \mathrm{s})\end{array}$ & $\begin{array}{c}\text { Acoustic impedance } \\
Z=\rho \cdot v_{L}\left(\mathrm{~N} \cdot \mathrm{s} / \mathrm{m}^{3}\right)\end{array}$ \\
\hline Air & 1.2 & 343 & 412 \\
\hline Cured resin & $\sim 1140$ & $\sim 2240$ & $\sim 2.55 \mathrm{e} 6$ \\
\hline
\end{tabular}

TABLE V: Material properties of air and cured resin of 3D printing. Due to the 6200 times higher acoustic impedance of resin, the air-resin interface can be treated as hard-wall boundary conditions in calculations. The pressure field inside the resin are ignored.

(a) Experiment setup A

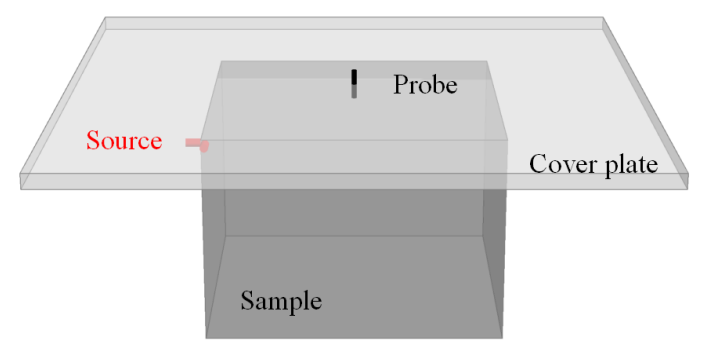

(c) Measured surface states in setup A

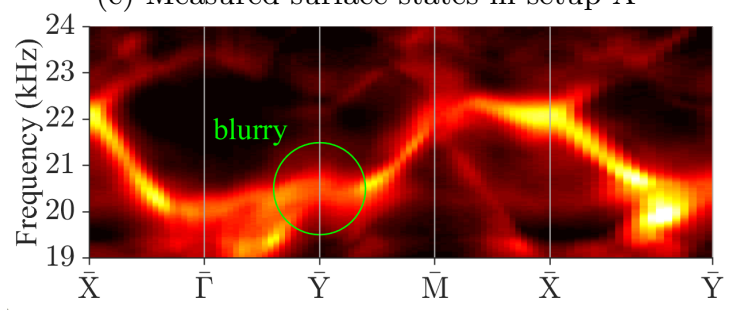

(e) Measured surface states in setup A

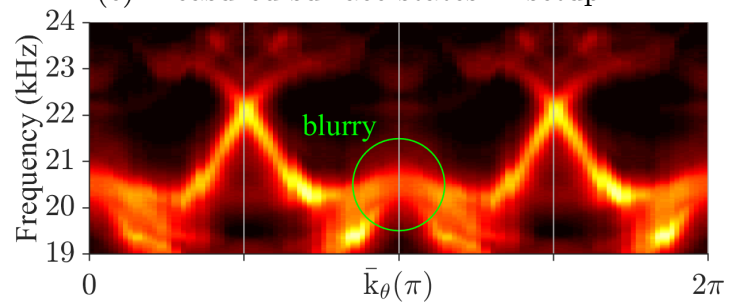

(b) Experiment setup B

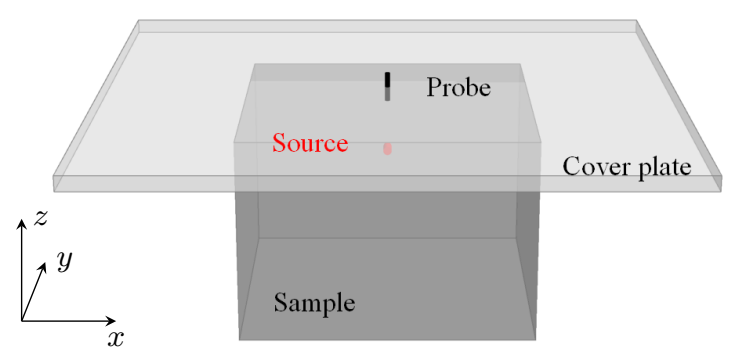

(d) Measured surface states in setup B

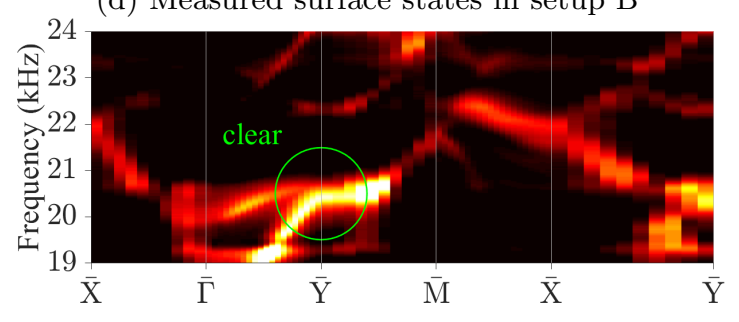

(f) Measured surface states in setup B

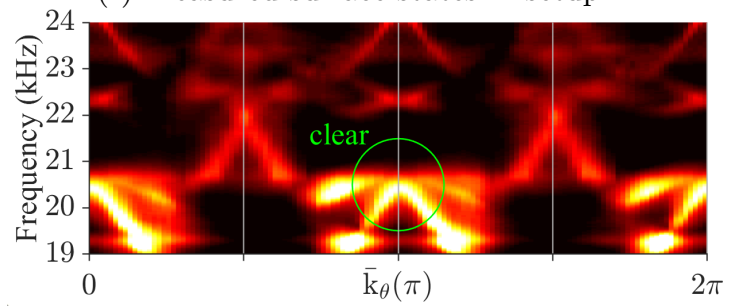

FIG. S5: Experimental data from different positions of the source excitations. (a) Setup A with the source fixed at the top corner of the sample, whose measurement results are shown in (c) and (e). (b) Setup B with the source fixed at the center of top edge of the sample, whose measurement results are shown in (d) and (f). 